\section{The direction of scratch test}

I read with interest the letter by Dr Motoi $e t$ $a l^{1}$ on the use of "direction of scratch" test in the assessment of posterior column dysfunction. They correlated the number of errors in the scratch test with central conduction times in somatosensory evoked potentials and with vibration perception thresholds, demonstrating correlation coefficients of 0.56 and 0.34 respectively $(p<0.01)$. This led them to conclude that the scratch test could be "recommended as a simple and yet reliable clinical neurological examination for detecting posterior column dysfunction".

In Hankey and Edis' original description of the sign, ${ }^{2}$ they defined a clearly abnormal test as 3 or more errors out of 10 . Applying this definition to Motoi et al's data for central conduction times, the predictive value for a positive test is only $60 \%$ (that is, $60 \%$ with an abnormal scratch test will have abnormal central conduction times). Conversely, the predictive value for a negative test is $80 \%$ (that is, $80 \%$ with a normal scratch test will have normal central conduction times). These figures cast some doubts on the usefulness of this test for clinical assessment of posterior column function.

Predictive values for the scratch test could not be calculated for vibration perception thresholds as Motoi et al did not provide a normal range. It would be of interest to have this information.

AJITH GOONETILLEKE Department of Neurology,
Westminister Hospital, London, UK

1 Motoi Y, Matsumoto H, Kaneshige Y, Chiba $S$. A reappraisal of "direction of scratch" test using somatosensory evoked potentials test using somatosensory evoked potentials and vibration perception. $\mathcal{F}$

2 Hankey GJ, Edis R. The utility of testing tactile perception of direction of scratch as sensitive clinical sign of posterior colum dysfunction in spinal cord disorders. f Neurol Neurosurg Psychiatry 1989;52 395-8.

\section{BOOK REVIEWS}

All titles reviewed here are available from the BMJ Bookshop, PO Box 295, London WC1H 9TE. Prices include postage in the United Kingdom and for members of the British Forcs Overseas, but overseas customers should add $£ 2$ per item for postage and packing. Payment can be made by cheque in sterling drawn on a United Kingdom bank, or by credit card (Mastercard, Visa or American Express) stating card number, expiratory date, and your full name.

NEUROMETHODS 22: Animal Models of Neurological Disease, II. Metabolic Encephalopathies and the Epilepsies. Edited by AA BOULTON, GB BAKER and RF BUTTERWORTH. (Pp 373; Price: £76.00). 1992. Published by Humana Press. UK Distrib: Chichester, J Wiley \& Sons Ltd. ISBN 0-89603-211-6.
Neuromethods 22 is the companion volume to Neuromethods 21 which had covered animal models of neurodegenerative diseases. The purpose and format of the current volume is identical to its partner in that experimental animal model systems of neurological diseases are discussed in both general and practical terms. Not only is the general style totally consistent with Neuromethods 21 , but the very high standard has been maintained. Indeed, I found this volume even more relevant to common clinical problems. Despite the title of the book, its scope is slightly wider since it covers animal models of human stroke and brain hypoxia, a variety of metabolic encephalopathies and also various models of epilepsy.

There are opening chapters on experimental models of human stroke and anima models of brain hypoxia. These subjects are obviously complimentary to some extent and both include detailed experimental protocols. Dr McCandless has co-authored al three excellent chapters on seizures (as well as the one on human stroke), and these include chemically induced models of seizures, the kindling model of epilepsy, and genetically based animal models of seizures Although kindling is a well-known phenomenon the precise mechanisms explaining this phenomenon are still not fully understood. These chapters are short but concise. There are a variety of chapters on metabolic animal models including those of the Wernicke-Korsakoff syndrome, hepatic encephalopathies, Reye's syndrome, NiacinNicotinamide deficiency, pyridoxine deficiency and a final chapter on hereditary hyperammonaemias. All are of considerable interest both for basic scientists and clinicians. In general the experimental detail of this volume is even greater than that of Neuromethods 21 . I was particularly struck by the chapter on the hepatic encephalopathies in which even the eight types of instruments and three types of suture materials are included! In general there were rather more illustrations and diagrams in this volume, which I welcomed.

Unquestionably this volume is every bit as good as its sister volume, and the two books together will serve as an invaluable source of general and practical information on experimental animal models of a variety of human neurological diseases. Despite its high price of $£ 76.00$ it is likely to be highly valued by neuroscientists.

PETER GE KENNEDY

Principles and Practice of Restorative Neurology. Edited by $R$ R YOUNG and P J DelWaide. (Pp 222; Price: $£ 40.00$ ). 1992. Oxford, Butterworth Heinemann. ISBN 0-7506-1172-3.

Recovery of function following a lesion in the central nervous system has always been an enigma to the Neurologist. The phenomenon is important because if the mechanisms of recovery were understood, not only would the mode of organization of the central nervous system be clearer, but opportunities for aiding this recovery would become more likely. In the 1960's the concept that following partial denervation the undamaged central nervous system would react in such a way that unused or little used pathways would become more effective was first suggested, on the basis of experimental studies, together with the corollary that part of the clinical picture seen after a lesion in the central nervous system was, in fact, due to the reaction of the intact central nervous system. This implied that the problem of rehabilitation of patients with chronic neurological defici should be via active "re-education" of the intact central nervous system and moved rehabilitation away from the management or treatment of complications which arise as a result of dysfunction.

The phenomena of sprouting of new synapses, unmasking of existing synapses, and alteration of receptive fields, have altered our view. By the late 1970's and early 1980's restorative neurology was established. There may be earlier claims for the coining of the term "restorative neurology" but it was Dimitrijevic who defined it as a branch of neurological sciences concerned with active procedures applied to the impaired nervous system in order to modify abnormal neuro-control. Since that time there has been an increasing interest in neuromodulation of the nervous system using physical or chemical stimulation and other methods to alter neurological function. The field is something of a departure from conventional neurology. Although very accurate diagnosis is essential, the emphasis in restorative neurology has always been on trying to assess the neurophysiological state as accurately as possible.

The interests encompass those of the basic scientist interested in plasticity of the nervous system, engineers involved in computer modelling, the Neurologist and Neurosurgeon involved in the function of the nervous system, the Anaesthetist with an interest in pain, and even the Vascular Surgeon and Cardiologist who sees the effect of neuromodulation outside the central nervous system.

In this excellent book, the editors Delwaide and Young define restorative neurology as a subspeciality of neurology dealing with "techniques and strategies used to restore a disordered nervous system to a state of optimal function" and they stress the wide ranging interests and specialities of the proponents, many of whom contribute to this volume. The editors, again, stress the importance of correct diagnosis and the importance of quantitative assessment.

There are chapters reviewing the epidemiology of disability, quantitative evaluation, biochemical changes after stroke and trauma, plasticity, pharmacotherapy, engineering aspects, principles of motor learning and training, as well as more conventional chapters on stroke, spinal cord injury, multiple sclerosis and Parkinson's disease. Newer technologies and disciplines of neurostimulation, surgical treatment of epilepsy and molecular genetics are summarized and the editors conclude with a thoughtful look to the future.

This book is strongly recommended. LS ILLIS

Plasticity in the Nervous System (Monographs in Neuroscience Series. Vol. 5). English Edition, Revised and Updated by BORIS I KOTLYAR. Translated and Edited by JOHN K YOUNG. (Pp 305; Price $\$ 56.00$, f30.00). 1992. Philadelphia, Gordon \& Breach Publishers. ISBN 2-88124-525-0. 
The title is honest and true, but will lead you astray. The author; Boris who? maybe you've heard of him. Then the declaration "Translated from the Russian by John $\mathrm{K}$ Young" gives you the clue. It is not just a translation of Russian work, but more a synthesis of Russian and western neurophysiology. There are about equal numbers of references to the Russian and to the western literature, the latter quaintly called "English" even though the odd one is in German. The late Professor Kotlyar was at the Department of Physiology of Higher Nervous Activity at Moscow State University and this book, highly acclaimed in Russia was completed in 1986, shortly before his death in 1990 .

Most of us in the west are aware that there is a fine tradition of neurophysiological work in Russia and the east but hardly any of us have read any of it in recent years. There are a few Russian journals included in index medicus, but most scientific papers are published in the proceedings of the many regional academies of science within the CIS. There is a Russian abstracting service, The All Union Institute for Scientific Information (INITI) which is probably available in this country through the national lending library, but unless you can read Russian it is not of much help.

It is therefore valuable for us to have a book like this written by an eminen Russian neuro-scientist who has achieved a valuable synthesis of Eastern and Western literature in this book of plasticity. Plasticity is a set of increasingly important processes and phenomena but where do they happen and what makes them happen? Herein lies the value of the book in exploring plasticity from the level of the synapse and subsynaptic membranes through neurones and reflexes to brains and behaviour. The book does not pretend to be encyclopaedic but each chapter provides a balanced synthesis of Russian and Western work. The influence of I P Pavlov, who died in 1936, is still strong; pavlovian reinforcement, inhibition, association and extinction are still with us but in a modified form more befitting to the age of the micro-electrode. You must remember that Pavlov used a salivary gland for recording nervous activity.

Brain "states" feature prominently. There is no definition of a state but it represents the particular relationship found between sets of neurones from time to time for instance arousal, attention and drowsiness are all behavioural manifestations of states. States cannot be studied by microelectrodes. Nevertheless these important concepts are often overlooked in Western neurophysiology. The sections on higher nervous activity bring to mind the works of Edelman and the theory neuronal group selection. One begins to feel that if Edelman knew his Pavlov as well as he knows Darwin then something really exciting might happen. Here perhaps lies a tragedy of the cold war. If Edelman and Kotlyar had been able to engage in a dialogue, a richer synthesis and a deeper understanding of processes might have been forthcoming: but they weren't and it is for future scientists to move along these lines. Kotlyar's thoughtful and scholarly views are nonetheless valuable.

This book draws back the iron curtain for us and allows us to see neuroscience on each side in a new light. The publishers, editors and translators and above all the authors of this and previous volumes are to be congratulated. It is to be hoped that literature, databases, abstracting and translating services pick up this initiative so that Eastern neuroscience can be assimilated along with that of the west, both sides will be enriched.

EM SEDGWICK

Neurocytopathology. By J M ANDREWS and G B SCHUMANN. 1992. (Pp 325 Illustrated; Price: £96.00). London, Williams \& Wilkins Ltd. ISBN 0-68300227-9.

This is a well thought out, practical and useful book. In a brief introductory chapter, the authors clearly define their aims: to present neurocytopathology in a concise and accessible format to surgical pathologists, cytologists and clinicians, to provide relevant information to enable the practice of the more important techniques and to give further guidance both for the literature and for sources of continuing medical education. The book fulfils these rather ambitious goals remarkably well. One chapter gives a brief overview of the cellular components and histological structures of the nervous system: this hardly amounts to more than a series of short vignettes and is perhaps the weakest part of the book, although it may serve as a guide to the uninitiated. There are no fewer than four chapters dealing with methodology: general aspects of CSF cytopathology, qualitative assessment of and quantitative approach to CSF cytopathology and cytological methods applied to neural tissue biopsies and aspirates. These chapters are full of useful practical information ranging from the actual descriptions of techniques to warning on artefacts.

The backbone of the book is the two chapters which give an account of diseases: one on tumours and related conditions, the other on non-neoplastic disorders. The former covers the more frequent entities and is, by no means, comprehensive. The latter include neurodegenerative, demyelinating, vascular, infectious and inflammatory diseases, leukodystrophies and the nonneoplastic disorders of the eye and orbit. A separate chapter is devoted to pitfalls, medico-legal and diagnostic, listing 10 of the former and 190 of latter. However, the greatest single asset of the book is the collection of more than 200 good quality, colour illustrations. I warmly recommend this book.

PL LANTOS

Pediatric Neuro-oncology /3 New Trends in Clinical Research (Monographs in Clinical Pediatrics Series). Edited by R J PACKER, W A BLEYER and C POCHEDLY. (Pp 320 Illustrated; Price: Not Indicated). 1992. New York, Harwood Academic Publishers. ISBN 3-7186-0524-4.

The purpose of this series is to present in relation to a specific theme-in this case tumours of the CNS in childhood-both authoritative clinical and basic science aspects and to attempt to correlate the two. In this aim the text is successful. The volume is a compilation of some of the topics covered at the International Symposium on Paediatric Neuro-Oncology help on 3 June 1989 in Seattle.

A strong plea is made in the preface that centralisation of care of children with CNS tumours continues and emphasises that effective management requires contributions from many paediatric sub-specialties including neurosurgery, neurology, oncology, radiotherapy, rehabilitation, psychology, endocrinology and psychiatry. Perhaps added to this list should be educationalists.

The volume covers issues of diagnosis, classification, surgical advances and chemotherapy. Specific clinical problems are discussed. An example would be the treatment of infants with brain tumours where most of what can be stated is particular difficulties rather than reporting advances.

Of particular interest is the discussion and presentation of the pathological data and the manner in which insight into the molecular phenotype of neural cells has improved understanding of the biology of brain tumours and systems of classification. Inadequacies of present systems for tumour classification based on presumed cell of origin and differentiation potential are discussed. There are excellent chapters on the investigation and clinical management of paediatric brain tumours in particular brain stem and spinal cord tumours in childhood and discussion of recent advances in management using hyperfractionated radiotherapy and high dose chemotherapy with autologous bone marrow rescue. For a comprehensive overview of the subject greater emphasis on such important issues as assessing and mitigating adverse effects of treatment would be necessary. Rehabilitation, the functioning of multidisciplinary management team, family support and audit of an overall service are not covered.

The volume can be recommended for those involved in the day to day management of paediatric brain tumours.

MA CLARKE

Tardive Dyskinesia: A Task Force Report of The American Psychiatric Association. By JOHN KANE (Pp 282; Price: £22.50). 1992. London, The Eurospan Group. ISBN 0-89042-230-3 (H/bk).

Prolonged exposure to neuroleptic drugs causing delayed or tardive dyskinesia has been generally accepted for over 20 years. This was deduced from the observed exacerbation or first appearance of dyskinesia with cessation of treatment, the suppression of dyskinesia with reintroduction of neuroleptic, and the slow improvement in some patients after extended drug withdrawal. However, the culpability of neuroleptics has recently been questioned by growing awareness of involuntary movements indistinguishable from tardive dyskinesia occurring in drug-naive psychotic patients and the possible involvement of basal ganglia dysfunction in psychosis. The authors of this report on tardive dyskinesia from the American Psychiatric Association state that in their view 'the consensus at the present time is that antipsychotic drugs do play a major role in producing, precipitating, or evoking abnormal involuntary movements'. The evidence is very suggestive but not compelling. 